\title{
Finite temperature dynamic susceptibility of the free Bose gas
}

\author{
F.Mazzanti ${ }^{1,2}$ and A.Polls ${ }^{3}$ \\ ${ }^{1}$ Institut für Theoretische Physik, Johannes Kepler Universität Linz, A-4040 Linz, Austria \\ ${ }^{2}$ Departament d'electrònica, Enginyeria i Arquitectura La Salle, \\ Pg. Bonanova 8, Universitat Ramon Llull, E-08022 Barcelona, Spain \\ ${ }^{3}$ Departament d'Estructura i Constituents de la Matèria, \\ Diagonal 645, Universitat de Barcelona, E-08028 Barcelona, Spain
}

October 30, 2018

\begin{abstract}
A detailed calculation of the real part of the finite temperature dynamic susceptibility of the free Bose gas is presented. After a short discussion on the different ways in which it can be calculated for temperatures above and below the Bose-Einstein transition temperature, its main properties and its evolution with $q$ and $T$ are analyzed. Finally, expressions for the lowest order energy weighted sum rules are also derived and studied.

PACS: 05.30.Fk, 61.12.Bt
\end{abstract}

KEYWORDS: Dynamic susceptibility, free Bose gas. 
The clear resolution of Bose-Einstein condensates in clouds of Alkali atoms at ultralow temperatures has revived the interest in the study of free and weakly interacting, homogeneous and inhomogeneous Bose systems during the last years [1]. Recent experimental evidence [2, 3] indicates that Bragg scattering can be succesfully applied to measure the dynamic structure function $S(q, \omega)$ of trapped Bose-Einstein condensates, and hence to directly probe their momentum distribution 4 .

During the last thirty years, much experimental and theoretical efforts have also been devoted towards the resolution of the condensate from neutron scattering in other, more correlated systems like pure ${ }^{4} \mathrm{He}$ and ${ }^{3} \mathrm{He}-{ }^{4} \mathrm{He}$ mixtures. However, none of the available formalisms have been able to accurately describe the influence of the temperature on the response, and particularly its influence on the condensate fraction, both at low and high momentum transfer [5].

In dilute systems, like the Bose-Einstein condensates of trapped alkali atoms, correlations are so weak that the response is expected to be qualitatively well described, at least at low momentum transfer, starting from the response of a free system and introducing the effect of the correlations through the Random Phase Approximation (RPA). In a previous work [6], a detailed description of the finite temperature dynamic structure function of the free Bose gas has been presented and discussed, focusing particularly on how $q$ and $T$ affect the total response and its coherent and incoherent parts. In this work we extend the analysis to the real part of the dynamic susceptibility $\chi(q, \omega ; T)$, which is the building block from which the RPA is constructed.

The dynamic susceptibility of an homogeneous free system is given by [0], 8]

$$
\begin{aligned}
\chi(q, \omega ; T) & =\frac{1}{N} \sum_{\mathbf{p}} n_{\mathbf{p}}\left[\frac{1}{\omega-t_{\mathbf{p}+\mathbf{q}}+t_{p}+i \eta}-\frac{1}{\omega-t_{p}+t_{\mathbf{p}-\mathbf{q}}+i \eta}\right] \\
& =\frac{\nu}{(2 \pi)^{3} \rho} \int d \mathbf{p} n(p)\left[\frac{1}{\omega-t_{\mathbf{p}+\mathbf{q}}+t_{p}+i \eta}-\frac{1}{\omega-t_{p}+t_{\mathbf{p}-\mathbf{q}}+i \eta}\right],
\end{aligned}
$$

where $\rho$ is the density, $t_{p}=p^{2} / 2 m$ is the free kinetic energy spectrum, $n(p)$ is the momentum distribution proportional to the occupation probability of each single-particle state of definite momentum, and $\nu$ is the spin-isospin degeneracy which will be taken equal to unity throughout this letter.

In the specific case of a free Bose gas case, $n(p)$ is the sum of a condensate and a non- 
condensate terms

$$
n(p)=(2 \pi)^{3} \rho n_{0}(T) \delta(\mathbf{p})+\frac{1}{z^{-1} e^{\beta p^{2} / 2 m}-1},
$$

where $\beta=1 / T$ is the inverse of the temperature and $z$ is the fugacity, related to the chemical potential $\mu$ and the temperature through $z=e^{\beta \mu}$. The condensate fraction value $n_{0}(T)$ varies with the temperature according to a $T^{3 / 2}$ law

$$
n_{0}(T)=1-\left(\frac{T}{T_{c}}\right)^{3 / 2}
$$

where $T_{c} \approx 3.31 \rho^{2 / 3} / m$ is the temperature at which the Bose-Einstein condensation sets in. Finally, the chemical potential $\mu(T)$ is fixed at each $T$ by imposing the particle normalization condition

$$
\frac{1}{(2 \pi)^{3} \rho} \int d \mathbf{p} n(p)=1
$$

At finite temperature, the imaginary part of the dynamic susceptibility is related to the dynamic structure function $S(q, \omega ; T)$ through the general relation [8]

$$
\operatorname{Im}[\chi(q, \omega ; T)]=-\pi[S(q, \omega ; T)-S(q,-\omega ; T)] \equiv-\pi\left(1-e^{-\beta \omega}\right) S(q, \omega ; T),
$$

where use has been made of the detailed balance condition, which relates the $\omega>0$ and the $\omega<0$ contributions to the finite temperature response in the form

$$
S(q,-\omega ; T)=e^{-\beta \omega} S(q, \omega ; T)
$$

The finite temperature $S(q, \omega ; T)$ of the free Bose gas has been calculated in [6] and yields, in terms of a new set of dimensionless variables that will be used throughout this work $\tilde{q}=q / k_{B}$, $\tilde{\omega}=\omega / \epsilon_{B}$ and $\tilde{T}=T / \epsilon_{B}$ where $\epsilon_{B}=k_{B}^{2} / 2 m$ and $k_{B}=\rho^{1 / 3}$ define the energy and momentum scales,

$$
\begin{aligned}
\tilde{S}(\tilde{q}, \tilde{\omega} ; \tilde{T})= & \frac{n_{0}(\tilde{T})}{1-e^{-\tilde{\omega} / \tilde{T}}}\left[\delta\left(\tilde{\omega}-\tilde{q}^{2}\right)-\delta\left(\tilde{\omega}+\tilde{q}^{2}\right)\right] \\
& -\frac{\tilde{T}}{16 \pi^{2} \tilde{q}} \frac{1}{\left(1-e^{-\tilde{\omega} / \tilde{T}}\right)} \ln \left[\frac{1-z e^{-(\tilde{\omega} / \tilde{q}-\tilde{q})^{2} / 4 \tilde{T}}}{1-z e^{-(\tilde{\omega} / \tilde{q}+\tilde{q})^{2} / 4 \tilde{T}}}\right] .
\end{aligned}
$$

The first term in this expression takes into account the contribution of the atoms in the condensate and appears in the form of two delta peaks centered at the quasielastic recoil energies $\tilde{\omega}= \pm \tilde{q}^{2}$. The second term describes the contribution coming from the rest of the atoms and 
shows itself as a function with two well defined peaks of finite width and height centered at the same energies.

The real part of the dynamic susceptibility splits also in two pieces, corresponding to the condensate and non-condensate contributions, respectively. The condensate part $\operatorname{Re}\left[\tilde{\chi}_{c}(\tilde{q}, \tilde{\omega} ; \tilde{T})\right]$ is equal to $n_{0}(T)$ times the real part of the $\tilde{T}=0$ dynamic susceptibility

$$
\operatorname{Re}\left[\tilde{\chi}_{c}(\tilde{q}, \tilde{\omega} ; \tilde{T})\right]=n_{0}(T) \operatorname{Re}\left[\frac{1}{\omega-\tilde{q}^{2}+i \eta}-\frac{1}{\omega+\tilde{q}^{2}+i \eta}\right]
$$

thus diverging at $\tilde{\omega}= \pm \tilde{q}^{2}$. On the other hand, the non-condensate part is a genuine contribution due to the finite temperature and is the main quantity analyzed in this work.

The real part of the dynamic susceptibility can be calculated in different ways, depending on whether the temperature is above or below the Bose-Einstein transition temperature. In a standard treatment, one can just evaluate it by using a Kramers-Kronig relation

$$
\operatorname{Re}[\tilde{\chi}(\tilde{q}, \tilde{\omega} ; \tilde{T})]=\frac{1}{\pi} \mathcal{P} \int d \tilde{\omega}^{\prime} \frac{\operatorname{Im}[\tilde{\chi}(\tilde{q}, \tilde{\omega} ; \tilde{T})]}{\tilde{\omega}^{\prime}-\tilde{\omega}},
$$

where $\mathcal{P}$ stands for Principal Value Integration. This expression relies on the implicit assumption that $\operatorname{Im}[\tilde{\chi}(\tilde{q}, \tilde{\omega} ; \tilde{T})]$ is an analytic function in the upper half of the complex plane, and that it decreases fast enough with increasing energy. Notice also that Eqs. (5) and (9) imply that $\operatorname{Im}[\tilde{\chi}(\tilde{q}, \tilde{\omega} ; \tilde{T})]$ and $\operatorname{Re}[\tilde{\chi}(\tilde{q}, \tilde{\omega} ; \tilde{T})]$ are odd and even functions of $\omega$, respectively.

Alternatively, one can evaluate $\operatorname{Re}[\tilde{\chi}(\tilde{q}, \tilde{\omega} ; \tilde{T})]$ as an infinite series, generalizing the result originally derived by Khanna and Glyde [9] for the free Fermi gas. Two different expressions are obtained in this way. In both cases, the dynamic susceptibility is written in the form

$$
\tilde{\chi}(\tilde{q}, \tilde{\omega} ; \tilde{T})=\tilde{\chi}(\tilde{q}, 0 ; \tilde{T})+\int_{0}^{\tilde{\omega}} d \xi \frac{\partial}{\partial \xi} \tilde{\chi}(\tilde{q}, \xi ; \tilde{T})
$$

where the partial derivative in the rhs can be evaluated from the definition in Eq. (1) and is expressed as an integral that may be carried out on the complex plane. As a result one gets an infinite series corresponding to a sum of residues, that has to be inserted in Eq. (10) and integrated to get the total dynamic susceptibility.

In a first approach, one integrates this series term by term to end up with

$$
\begin{aligned}
\operatorname{Re}[\tilde{\chi}(\tilde{q}, \tilde{\omega} ; \tilde{T})] & =\operatorname{Re}[\tilde{\chi}(\tilde{q}, 0 ; \tilde{T})] \\
& +\frac{\tilde{T}}{8 \pi \tilde{q}} \sum_{n}\left[\tan ^{-1}\left(\frac{2 \tilde{y}_{1} \tilde{b}_{n}}{\tilde{y}_{1}^{2}-\left(\tilde{a}_{n}^{2}+\tilde{b}_{n}^{2}\right)}\right)-\tan ^{-1}\left(\frac{2 \tilde{y}_{2} \tilde{b}_{n}}{\tilde{y}_{2}^{2}-\left(\tilde{a}_{n}^{2}+\tilde{b}_{n}^{2}\right)}\right)\right],
\end{aligned}
$$


where the index $n$ runs from 0 to $\infty$. In Eq. (11), $\tilde{a}_{n}$ and $\tilde{b}_{n}$ are the real and imaginary parts of the poles of the momentum distribution laying in the first quadrant

$$
\begin{aligned}
& \tilde{a}_{n}=\frac{1}{\sqrt{2}}\left[\tilde{\mu}+\left(\tilde{\mu}^{2}+4 \pi^{2} n^{2} \tilde{T}^{2}\right)^{1 / 2}\right]^{1 / 2} \\
& \tilde{b}_{n}=\frac{1}{\sqrt{2}}\left[-\tilde{\mu}+\left(\tilde{\mu}^{2}+4 \pi^{2} n^{2} \tilde{T}^{2}\right)^{1 / 2}\right]^{1 / 2},
\end{aligned}
$$

while $\tilde{y}_{1}$ and $\tilde{y}_{2}$ are the usual West scaling variables, related to the momentum and the energy transfer through

$$
\tilde{y}_{1}=\frac{1}{2}\left(\frac{\tilde{\omega}}{\tilde{q}}-\tilde{q}\right) \quad \& \quad \tilde{y}_{2}=\frac{1}{2}\left(\frac{\tilde{\omega}}{\tilde{q}}+\tilde{q}\right) .
$$

Alternatively, one can first evaluate the sum of all the residues contributing to the energy derivative of the real part of the susceptibility

$$
\begin{aligned}
\frac{\partial}{\partial \tilde{\omega}} \operatorname{Re}[\tilde{\chi}(\tilde{q}, \tilde{\omega} ; \tilde{T})]= & \frac{\tilde{T}}{8 \pi \tilde{q}^{2}} \sum_{n>0}\left\{2 \pi n \tilde{T}\left(\frac{\tilde{a}_{n}}{\tilde{a}_{n}^{2}+\tilde{b}_{n}^{2}}\right)\left[\frac{\tilde{y}_{2}^{2}}{\left(\tilde{y}_{2}^{2}-\tilde{\mu}\right)^{2}+4 \pi^{2} \tilde{T}^{2} n^{2}}-\frac{\tilde{y}_{1}^{2}}{\left(\tilde{y}_{1}^{2}-\tilde{\mu}\right)^{2}+4 \pi^{2} \tilde{T}^{2} n^{2}}\right]\right. \\
& \left.-\left(\frac{\tilde{b}_{n}}{\tilde{a}_{n}^{2}+\tilde{b}_{n}^{2}}\right)\left[\frac{\tilde{y}_{2}^{2}\left(\tilde{y}_{2}^{2}-\tilde{\mu}\right)}{\left(\tilde{y}_{2}^{2}-\tilde{\mu}\right)^{2}+4 \pi^{2} \tilde{T}^{2} n^{2}}-\frac{\tilde{y}_{1}^{2}\left(\tilde{y}_{1}^{2}-\tilde{\mu}\right)}{\left(\tilde{y}_{1}^{2}-\tilde{\mu}\right)^{2}+4 \pi^{2} \tilde{T}^{2} n^{2}}\right]\right\} .
\end{aligned}
$$

and afterwards perform the integration. Although this method seems to be more elaborated than the simple sum in Eq. (11), it is usually preferred since the former expression is known to converge rather slowly. In both cases, however, $\operatorname{Re}[\tilde{\chi}(\tilde{q}, 0 ; \tilde{T})]$ is a required quantity that can be obtained, proceeding as before, in the form of a series

$$
\begin{aligned}
\operatorname{Re}[\tilde{\chi}(\tilde{q}, 0 ; \tilde{T})]= & \operatorname{Re}[\tilde{\chi}(0,0 ; \tilde{T})] \\
& -\frac{\tilde{T}}{32 \pi \tilde{q}} \int_{0}^{\tilde{q}} d \tilde{k} \tilde{k}^{2} \sum_{n} \frac{\tilde{a}_{n} 2 \pi n \tilde{T}-\tilde{b}_{n}\left(\frac{\tilde{k}^{2}}{4}-\tilde{\mu}\right)}{\left(\tilde{a}_{n}^{2}+\tilde{b}_{n}^{2}\right)\left(\left(\frac{\tilde{k}^{2}}{4}-\tilde{\mu}\right)^{2}+4 \pi^{2} \tilde{T}^{2} n^{2}\right)}
\end{aligned}
$$

where

$$
\operatorname{Re}[\tilde{\chi}(0,0 ; \tilde{T})]=-\frac{1}{2 \pi^{2}} \int_{0}^{\infty} d \tilde{p} n(\tilde{p}) .
$$

and the sum extending from 0 to $\infty$ with $\tilde{a}_{n}$ and $\tilde{b}_{n}$ given in Eq. (12).

Finally, $\operatorname{Re}[\tilde{\chi}(\tilde{q}, \tilde{\omega} ; \tilde{T})]$ can also be evaluated by direct numerical integration of Eq. (1). After simple manipulations, one finds

$$
\begin{aligned}
\operatorname{Re}[\tilde{\chi}(\tilde{q}, \tilde{\omega} ; \tilde{T})] & =\frac{1}{8 \pi \tilde{q}} \operatorname{Re} \int d \tilde{p} \tilde{p} n(\tilde{p})\left[\ln \left(\frac{\tilde{y}_{2}-\tilde{p}+i \epsilon}{\tilde{y}_{2}+\tilde{p}+i \epsilon}\right)-\ln \left(\frac{\tilde{y}_{1}-\tilde{p}+i \epsilon}{\tilde{y}_{1}+\tilde{p}+i \epsilon}\right)\right] \\
& =\frac{1}{8 \pi \tilde{q}} \mathcal{P} \int_{0}^{\infty} d \tilde{p} \tilde{p} n(\tilde{p})\left[\ln \left|\frac{\tilde{y}_{2}-\tilde{p}}{\tilde{y}_{2}+\tilde{p}}\right|-\ln \left|\frac{\tilde{y}_{1}-\tilde{p}}{\tilde{y}_{1}+\tilde{p}}\right|\right]
\end{aligned}
$$


which can be safely carried out since their singularities are of the integrable type.

For temperatures above $\tilde{T}_{c}$, all three methods are equivalent and any of them can be used to obtain the non-condensate contribution to the real part of the dynamic susceptibility. Below $\tilde{T}_{c}$, however, only the direct integration method and the Kramers-Kronig relation can be applied, even though in the latter case the analyticity condition required for $\operatorname{Im}[\tilde{\chi}(\tilde{q}, \tilde{\omega} ; \tilde{T})]$ is clearly violated since at those temperatures the chemical potential vanishes and the fugacity $z$ goes to 1 , therefore the function presents two singularities on the real axis located at the quasielastic energies $\tilde{\omega}= \pm \tilde{q}^{2}$. However, these are logarithmic singularities that can be integrated on the complex plane and yield no additional contribution to the integrals. On the other hand, none of the series methods mentioned above can be used because below $\tilde{T}_{c}$ the momentum distribution grows as $\tilde{p}^{-2}$ for small $\tilde{p}$, thus making $\operatorname{Re}[\tilde{\chi}(0,0 ; \tilde{T})]$ in Eq. (16) diverge.

Since $\tilde{\mu}\left(\tilde{T} \leq \tilde{T}_{c}\right)=0$, the temperature dependence of $\operatorname{Re}\left[\tilde{\chi}\left(\tilde{q}, \tilde{\omega} ; \tilde{T} \leq \tilde{T}_{c}\right)\right]$ can be easily extracted once it is written in terms of two new variables $Q=\tilde{q} / \sqrt{\tilde{T}}$ and $\nu=\tilde{\omega} / \tilde{T}$. Moreover, one can define a new function

$$
\operatorname{Re}[\hat{\chi}(Q, \nu)] \equiv \frac{1}{\sqrt{\tilde{T}}} \operatorname{Re}[\chi(\tilde{q}, \tilde{\omega} ; \tilde{T})]
$$

that does not depend on the temperature when $Q$ and $\nu$ are taken as the new independent variables. Actually, this scaling property is also satisfied by the imaginary part of $\tilde{\chi}(\tilde{q}, \tilde{\omega}, \tilde{T})$ which inherits it from the $\tilde{T} \leq \tilde{T}_{c}$ behaviour of the dynamic structure function of the free Bose gas [6].

$\operatorname{Re}[\hat{\chi}(Q, \nu)]$ is shown in Fig. (11) for several values of $Q$. At low $\nu, \operatorname{Re}[\hat{\chi}(Q, \nu)]$ is negative and discontinuous at $\nu=Q^{2}$, jumping to positive values and afterwards decaying to 0 . The discontinuity at $\nu=Q^{2}$ is a direct consequence of the divergent behaviour of the momentum distribution at low momenta, and is therefore directly related to the presence of a Bose condensate. On the other hand, the decay of $\operatorname{Re}[\hat{\chi}(Q, \nu)]$ at large energies is of the form $\left(1-n_{0}(T)\right) 2 Q^{2} / \nu^{2} \tilde{T}^{3 / 2} \equiv 2 Q^{2} / \nu^{2} \tilde{T}_{c}^{3 / 2}$. This last property is mirrored from the fact that the real part of the dynamic susceptibility is related to the dynamic structure function through Eqs. (9) and (5), and this together with the $f$-sum rule satisfied by $\tilde{S}(\tilde{q}, \tilde{\omega} ; \tilde{T})$ is enough to determine the analytical behaviour of the real part of the susceptibility at large energies [8]. Finally, the factor $1-n_{0}(T)$ reflects the fact that we are only looking at the non-condensate contribution to the susceptibility, while the condensate term adds the extra strength required to have a total 
decay of the form $2 \tilde{q}^{2} / \tilde{\omega}^{2}$ as expected.

With rising momentum transfer the discontinuity shifts to higher $Q$ 's, the strength at low energies and around $Q^{2}$ is depressed, but the energy range where $\operatorname{Re}[\hat{\chi}(Q, \nu)]$ presents a significant contribution is increased. Due to the scaling in $Q$ and $\nu$, the effect of fixing $\tilde{T}$ and rising $\tilde{q}$ is equivalent to the effect of fixing $\tilde{q}$ and decreasing $\tilde{T}$. Therefore, the evolution with $\tilde{T}$ of $\operatorname{Re}[\tilde{\chi}(\tilde{q}, \tilde{\omega} ; \tilde{T})]$ at fixed $\tilde{q}$ can be read in the figure by moving from higher to lower $Q$ 's, while the evolution with $\tilde{q}$ at fixed $\tilde{T}$ is represented by the same sequence of curves but in reverse order. At $\tilde{T}=0$ the non-condensate contribution to the dynamic susceptibility vanishes and only the condensate term is left.

When $\tilde{T}$ crosses the Bose-Einstein transition temperature, the discontinuity at $\tilde{\omega}=\tilde{q}^{2}$ is smeared out and $\operatorname{Re}[\tilde{\chi}(\tilde{q}, \tilde{\omega} ; \tilde{T})]$ becomes a continuous function of $\tilde{\omega}$, as shown in Fig. (2) for a momentum transfer of $\tilde{q}=1$. This is due to the fact that, above $\tilde{T}_{c}$, the chemical potential is no longer 0 and thus the $\tilde{p} \rightarrow 0$ divergence in the momentum distribution is removed. Actually the process is quite fast, and already at $\tilde{T}=7$ and a momentum transfer of $\tilde{q}=1$, no apparent trace of a discontinuity in the real part of the dynamic susceptibility is left.

The evolution with $\tilde{T}$ of the non-condensate contribution to the real part of the dynamic susceptibility for $\tilde{T}>\tilde{T}_{c}$ and for three values of the momentum transfer is sketched in Fig. (3). The actual function depicted is $\tilde{T} \operatorname{Re}[\tilde{\chi}(\tilde{q}, \tilde{Y} ; \tilde{T})]$ with $\tilde{Y}=(\nu / Q-Q) / 2$, since in the high temperature limit the susceptibility of the free Bose gas approaches the $\tilde{T} \rightarrow \infty$ classical prediction which for energies around the quasielastic peak $\tilde{\omega}=\tilde{q}^{2}$ can be casted in the form

$$
\tilde{T} \operatorname{Re}\left[\tilde{\chi}_{c l}(\tilde{q}, \tilde{Y} ; \tilde{T} \rightarrow \infty)\right]=-\frac{1}{\sqrt{\pi}} \mathcal{P} \int_{-\infty}^{\infty} d z \frac{z e^{-z^{2}}}{z-\tilde{Y}},
$$

thus being a function of $\tilde{Y}$ alone. Since $\operatorname{Re}[\tilde{\chi}(\tilde{q}, \tilde{\omega} ; \tilde{T})]$ is and even function of $\tilde{\omega}$, only the contribution at positive energies is shown, so the initial point in each curve is $\tilde{Y}=-Q / 2$. As it can be seen from the figure, the departure from the classical limit at low temperatures is sizeable, while the Bose prediction gets closer to the classical one when the temperature is risen, as expected. However, the way in which the classical limit is reached depends on the momentum transfer. At low $\tilde{q}$, the low temperature susceptibility rapidly varies from negative values below the classical prediction to positive ones above it. With increasing momentum transfer, the strength of the Bose function at fixed temperature is progressively reduced, but the range of maximum variation in which the function goes from negative to positive values 
grows. In all cases, however, the large $\tilde{Y}$ tails of the Bose function conform to the classical prediction at any temperature, a fact that should not surprise since as commented above the dynamic susceptibility is known to decrease as $2 \tilde{q}^{2} / \tilde{\omega}^{2}$ independently of the temperature and the statistics.

The behaviour of the real part of the dynamic susceptibility at fixed $\tilde{T}$ larger than $\tilde{T}_{c}$ and for a sequence of increasing values of the momentum transfer $\tilde{q}$ is depicted in Fig. (44). In this case, the $\tilde{q} \rightarrow \infty$ limit of $\operatorname{Re}[\tilde{\chi}(\tilde{q}, \tilde{Y} ; \tilde{T})]$ at finite $\tilde{Y}$ can be obtained from the leading term in a $1 / \tilde{q}$ expansion, a property that is inherited from the high $\tilde{q}$ behaviour of the dynamic structure function [10]. As in the latter case and for the free Bose gas [6], the function

$$
\frac{\tilde{q}}{\tilde{T}} \operatorname{Re}[\tilde{\chi}(\tilde{q}, \tilde{Y} ; \tilde{T})]
$$

approaches a fixed curve when $\tilde{q} \rightarrow \infty$ that depends on the temperature only through the chemical potential. This limiting function is represented with a solid line in the figure, while the other curves show the way in which this limit is approached with increasing $\tilde{q}$. Notice that, contrary to what happened in the previous case, increasing $\tilde{q}$ at fixed $\tilde{T}$ makes the initial point $\tilde{Y}=-Q / 2$ in the limiting curve go to $-\infty$, thus emphasizing that the region of maximal variation of $\operatorname{Re}[\tilde{\chi}(\tilde{q}, \tilde{Y} ; \tilde{T})]$ lies always around $\tilde{Y} \approx 0$ corresponding to energies close to the quasielastic peak $\omega=\tilde{q}^{2}$. Notice also that the limiting curve is always reached from the right, as $(\tilde{q} / \tilde{T}) \operatorname{Re}[\tilde{\chi}(\tilde{q}, \tilde{Y} ; \tilde{T})]$ shifts to lower values of $\tilde{Y}$ when $\tilde{q}$ is risen.

The dependence of the real part of the susceptibility on $\tilde{T}$ and $\tilde{q}$ can also be analyzed by looking at the sum rules it satisfies, which are defined as the energy-weighted integrals

$$
\tilde{m}_{n}(\tilde{q} ; \tilde{T})=\int_{0}^{\infty} d \tilde{\omega} \tilde{\omega}^{n} \operatorname{Re}[\tilde{\chi}(\tilde{q}, \tilde{\omega} ; \tilde{T})] .
$$

Sum rules could also be defined extending the integration range to $\tilde{\omega} \in(-\infty, \infty)$, but then all the odd order ones would trivially vanish because $\operatorname{Re}[\tilde{\chi}(\tilde{q}, \tilde{\omega} ; \tilde{T})]$ is an even function of $\tilde{\omega}$. With the previous definition, and taking into account that the real part of the dynamic susceptibility decreases as $2 \tilde{q}^{2} / \tilde{\omega}^{2}$ at large energies, one readily notices that no sum rule with $n>0$ exists. However, an expansion around $\tilde{\omega}^{-1}=0$ displays a next-to-leading term of order $\tilde{\omega}^{-4}$, so one can still find a second energy weighted sum rule by subtracting the leading contribution at high energies. Proceeding in this way one finds the following relations

$$
\tilde{m}_{0}(\tilde{q} ; \tilde{T})=\int_{0}^{\infty} d \tilde{\omega} \operatorname{Re}[\tilde{\chi}(\tilde{q}, \tilde{\omega} ; \tilde{T})]=0
$$




$$
\tilde{m}_{2}(\tilde{q} ; \tilde{T})=\int_{0}^{\infty} d \tilde{\omega} \tilde{\omega}^{2}\left(\operatorname{Re}[\chi(\tilde{q}, \tilde{\omega} ; \tilde{T})]-\frac{2 \tilde{q}^{2}}{\tilde{\omega}^{2}}\right)=0 .
$$

These results actually apply to the total real part of the dynamic susceptibility, including the condensate contribution. However, they are general and therefore hold also for the $\tilde{T}=0$ susceptibility of the free Bose gas also. The condensate contribution at finite temperature is equal to $n_{0}(\tilde{T}) \operatorname{Re}[\tilde{\chi}(\tilde{q}, \tilde{\omega} ; \tilde{T}=0)]$, and thus its $\tilde{m}_{0}(\tilde{q} ; \tilde{T})$ and $\tilde{m}_{2}(\tilde{q} ; \tilde{T})$ also vanish. In this way, therefore, the above sum rules apply separately to the condensate and non-condensate parts of the dynamic susceptibility, respectively. Additionally, the fact that both sum rules vanish indicates that $\operatorname{Re}[\tilde{\chi}(\tilde{q}, \tilde{\omega} ; \tilde{T})]$ must have different regions of positive and negative values, a feature that is in fact general to all systems since the previous sum rules apply to both correlated and uncorrelated systems at $\tilde{T}=0$ and finite temperature.

Although the previous results hold for any system conserving the total number of particles, it is difficult to obtain generic expressions for higher order sum rules since then additional terms in the $\tilde{\omega}^{-1}$ expansion of $\operatorname{Re}[\tilde{\chi}(\tilde{q}, \tilde{\omega} ; \tilde{T})]$ must be subtracted, and the coefficients of this expansion are related to sum rules of the dynamic structure function $\tilde{S}(\tilde{q}, \tilde{\omega} ; \tilde{T})$, which are specific to each system. Furthermore, the simple subtraction of $2 \tilde{q}^{2} / \tilde{\omega}^{2}$ does not allow for the derivation of a $\tilde{m}_{1}(\tilde{q} ; \tilde{T})$ sum rule, a problem that remains even when higher orders in the $\tilde{\omega}^{-1}$ series expansion of $\operatorname{Re}[\tilde{\chi}(\tilde{q}, \tilde{\omega} ; \tilde{T})]$ are subtracted.

An alternative set of sum rules, valid for correlated Bose systems at $\tilde{T}=0$ and $\tilde{T}>0$ and for the noninteracting Bose gas at $\tilde{T}>0$, can be derived by subtracting to $\tilde{\chi}(\tilde{q}, \tilde{\omega} ; \tilde{T})$ the $\tilde{T}=0$ dynamic susceptibility $\tilde{\chi}^{0}(\tilde{q}, \tilde{\omega} ; 0)$ of the free Bose gas. As long as the real part is concerned, these become

$$
\tilde{M}_{n}(\tilde{q}, \tilde{T})=\int_{0}^{\infty} d \tilde{\omega} \tilde{\omega}^{n}\left(\operatorname{Re}[\tilde{\chi}(\tilde{q}, \tilde{\omega} ; \tilde{T})]-\operatorname{Re}\left[\tilde{\chi} \tilde{\chi}^{0}(\tilde{q}, \tilde{\omega} ; 0)\right]\right) .
$$

Using the explicit representation of the dynamic susceptibility in terms of the dynamic structure function, the first $\tilde{M}_{n}(\tilde{q}, \tilde{T})$ moments can be easily derived and yield

$$
\begin{aligned}
& \tilde{M}_{0}(\tilde{q} ; \tilde{T})=0 \\
& \tilde{M}_{1}(\tilde{q} ; \tilde{T})=-\int_{-\infty}^{\infty} d \tilde{\omega} \tilde{S}(\tilde{q}, \tilde{\omega} ; \tilde{T}) \ln \left(\frac{\tilde{\omega}^{2}}{\tilde{q}^{4}}\right), \\
& \tilde{M}_{2}(\tilde{q} ; \tilde{T})=0
\end{aligned}
$$

Interestingly enough, the expression of $\tilde{M}_{1}(\tilde{q} ; \tilde{T})$ indicates that even though it is not know a 
priori whether the dynamic structure function $\tilde{S}(\tilde{q}, \tilde{\omega} ; \tilde{T})$ of a given system does have sum rules to all orders [11], still some combinations of them may exist. Notice also that this sum rule is trivially satisfied by the condensate term, since the condensate contribution to $\tilde{S}(\tilde{q}, \tilde{\omega} ; \tilde{T})$ is a sum of two delta peaks centered at $\tilde{\omega}= \pm \tilde{q}^{2}$ where the logarithm cancels. Therefore and for the free Bose gas, the non-trivial part of $\tilde{M}_{1}(\tilde{q} ; \tilde{T})$ must be satisfied by the non-condensate term alone. Moreover and from the same argument given above, it is also apparent that the total set of sum rules must be separately satisfied by the condensate and non-condensate contributions to $\operatorname{Re}[\tilde{\chi}(\tilde{q}, \tilde{\omega} ; \tilde{T})]$. Finally, the zeroth and second order sum rules $\tilde{M}_{0}(\tilde{q} ; \tilde{T})$ and $\tilde{M}_{2}(\tilde{q} ; \tilde{T})$ are a direct consequence of the results shown in Eqs. (22) and (23).

The fact that below $\tilde{T}_{c}$ the temperature dependence of both the dynamic susceptibility and the response function can be extracted as indicated in Eq. (18) implies that an energy weighted sum rule can also be defined in the form

$$
\hat{\mathrm{M}}_{1}(Q ; \tilde{T})=\frac{1}{\tilde{T}^{3 / 2}} \tilde{M}_{1}(q ; \tilde{T}) \equiv \int_{-\infty}^{\infty} d \nu \tilde{S}(Q, \nu ; \tilde{T}) \ln \left(\frac{\nu^{2}}{Q^{4}}\right)
$$

where $\tilde{S}(Q ; \nu ; \tilde{T})=\tilde{S}_{n c}(\tilde{q}, \tilde{\omega} ; \tilde{T}) / \sqrt{\tilde{T}}$ is the non-condensate contribution to the response of the free Bose gas written in the variables $Q$ and $\nu$. Below the Bose-Einstein transition temperature, this factorization removes any dependence on $\tilde{T}$ and thus $\hat{\mathrm{M}}_{1}$ becomes a function of $Q$ alone. Above $\tilde{T}_{c}$, however, a dependence on $\tilde{T}$ remains through the chemical potential $\tilde{\mu}(\tilde{T}) . \hat{\mathrm{M}}_{1}(Q ; \tilde{T})$ is shown in Fig. (5) for several temperatures below and above $\tilde{T}_{c}$. In all cases, the low $Q$ behaviour is divergent, a feature produced by the $Q^{4}$ term inside the logarithm. On the other hand, the high $Q$ behaviour is dominated by a slowly decreasing tail that approach 0 from below. In the intermediate region, $\hat{\mathrm{M}}_{1}(Q ; \tilde{T})$ presents a minimum at some $Q$ that increases with the temperature, even though it is hardly noticeable at high $\tilde{T}$ 's.

In summary, it has been shown that the non-condensate contribution to the real part of the dynamic susceptibility of the free Bose gas at finite temperature can be evaluated in three different ways: by direct integration, using a Kramers-Kronig relation and performing a Khanna and Glyde -like series expansion. While the first two methods can be used above and below the Bose-Einstein transition temperature, the latter fails below $\tilde{T}_{c}$ since at those temperatures the starting point in the series diverges.

$\operatorname{Re}\left[\tilde{\chi}\left(\tilde{q}, \tilde{\omega} ; \tilde{T} \leq \tilde{T}_{c}\right)\right]$ is shown to scale in two new variables $Q=\tilde{q} / \sqrt{\tilde{T}}$ and $\nu=\tilde{\omega} / \tilde{T}$ from where its temperature dependence can be extracted, a condition that is lost above $\tilde{T}_{c}$ due to the 
finite value taken by the chemical potential $\tilde{\mu}(\tilde{T})$. Below $\tilde{T}_{c}$, the real part of the non-condensate contribution to the dynamic susceptibility presents a discontinuity at $\tilde{\omega}= \pm \tilde{q}^{2}$ produced by the singular behaviour of the momentum distribution at $\tilde{p} \rightarrow 0$. When $\tilde{T}$ exceeds $\tilde{T}_{c}$ this singularity in $n(\tilde{p} \rightarrow 0)$ is removed and as a result the previous discontinuity is smeared out. At high $\tilde{T}>\tilde{T}_{c}, \operatorname{Re}[\tilde{\chi}(\tilde{q}, \tilde{\omega} ; \tilde{T})]$ approaches the classical prediction computed from a Maxwell-Boltzmann momentum distribution which for energies close to the quasielastic peak $\tilde{\omega}=\tilde{q}^{2}$ can be brought to a form that scales in a single variable $\tilde{Y}=(\tilde{\omega} / \tilde{q}-\tilde{q}) / 2 \sqrt{\tilde{T}}$. On the other hand, the leading contribution to the high $\tilde{q}$ limit of $\operatorname{Re}[\tilde{\chi}(\tilde{q}, \tilde{\omega} ; \tilde{T})]$ can also be expressed in terms of $\tilde{Y}$ alone, a property that is inherited from the large momentum transfer behaviour of $\tilde{S}(\tilde{q}, \tilde{\omega} ; \tilde{T})$.

Low order energy weighted sum rules of the non-condensate contribution to the real part of the dynamic susceptibility are also derived and discussed. Since the long energy tails of $\operatorname{Re}[\tilde{\chi}(\tilde{q}, \tilde{\omega} ; \tilde{T})]$ decrease as $1 / \tilde{\omega}^{2}$, no sum rule of order higher than 0 exists. However, a new set of sum rules, obtained by subtracting the $\tilde{T}=0$ dynamic susceptibility of the free Bose gas, is proposed and analyzed. These sum rules are general and apply to any system conserving the total number of particles, and their analysis show that in general the real part of the susceptibility must have different regions where it changes sign. Despite the simplicity of the model analyzed, the noninteracting Bose gas, some of the conclusions drawn here are expected to enlighten some aspects of the finite temperature dynamic susceptibility in weakly interacting systems, which can be built starting from the non-interacting case in the framework of the Random Phase Approximation.

\section{Acknowledgments}

This work has been partially supported by the Austrian Science Fund under grant No. P12832-TPH, DGICYT (Spain) grant No. PB98-1247 and the program SGR98-11 from Generalitat de Catalunya. One of us wants to thank D. Mazzanti for giving a strong motivation to carry out this work. 


\section{References}

[1] F. Dalfovo, S. Giorgini, L. P. Pitaevskii, and S. Stringari, Rev. Mod. Phys. 71 ,463 (1999).

[2] J.Stenger, S.Inouye, A.P.Chikkatur, D.M.Stamper-Kurn, D.E.Pritchard, and W.Ketterle, Phys.Rev.Lett. 82, 4569 (1999).

[3] D.M.Stamper-Kurn, A.P.Chikkatur, A.Görlitz, S.Inouye, S.Gupta, D.E.Pritchard, and W.Ketterle, Phys.Rev.Lett. 83 (1999).

[4] F. Zambelli, L. Pitaevskii, D.M. Stamper-Kurn, and S. Stringari, Phys. Rev. A61,063608 (2000).

[5] H.R.Glyde, Excitations in Solid and Liquid Helium, Clarendon Press, Oxford (1994) and references therein.

[6] F.Mazzanti and A.Polls, Phys.Lett.A 275 (2000) 1.

[7] A.L.Fetter and J.D.Walecka, Quantum Theory of Many-Particle Systems, , McGraw-Hill, New York (1971).

[8] D.Pines and P.Nozières, The Theory of Quantum Liquids, Vol.I, Addison-Wesley, New York (1989).

[9] F.C.Khanna and H.R.Glyde, Can.J.Phys. 54, 648 (1976).

[10] H.A.Gersch, L.J.Rodriguez and P.N.Smith, Phys. Rev. A 5, 1547 (1972).

[11] A. Holas, in Strongly Coupled Plasma Physics, edited by F. J. Rogers and H. E. DeWitt (Plenum Press, New York, 1986), pp. 463-482. 


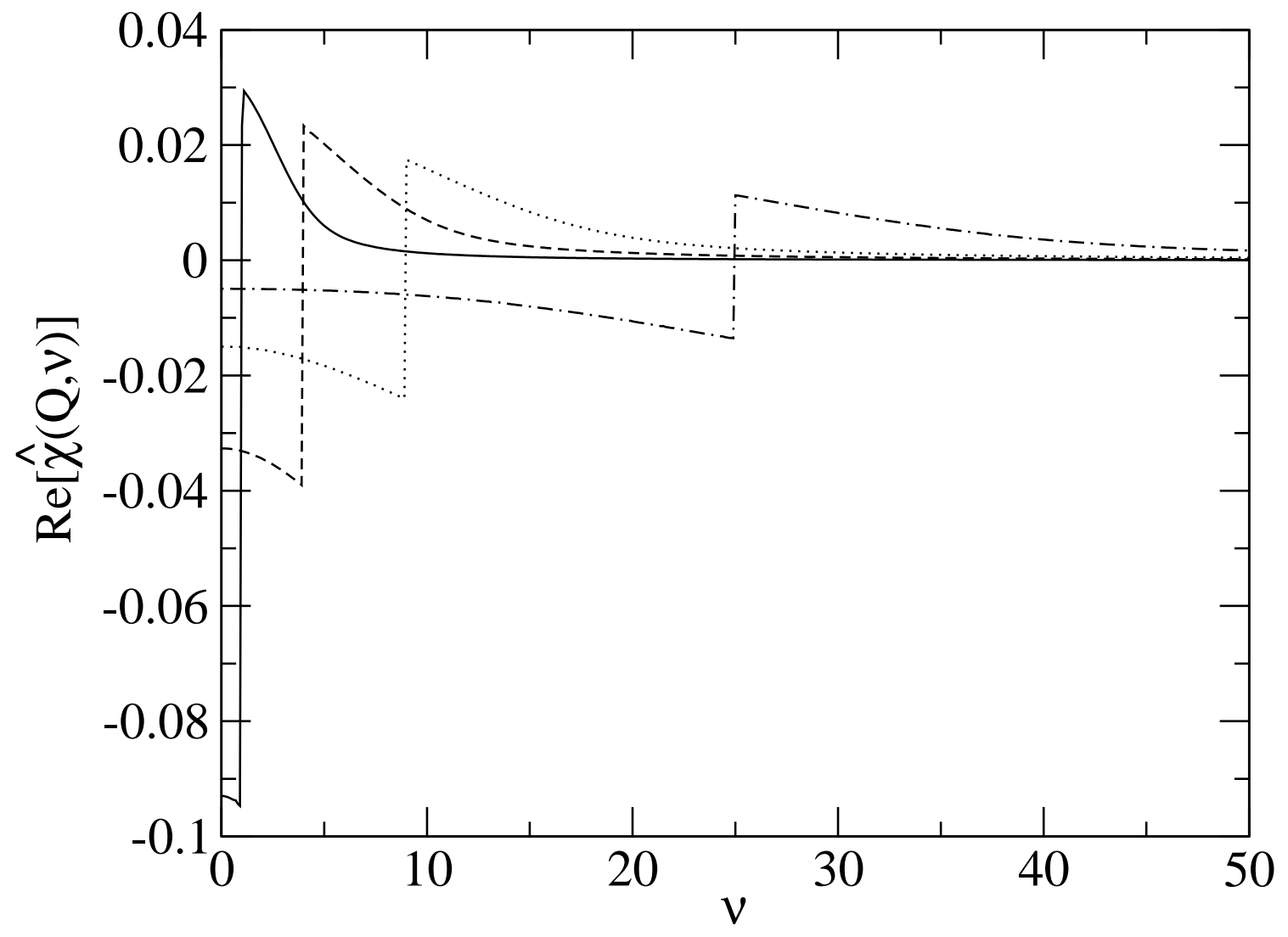

Figure 1: Real part of $\hat{\chi}(Q, \nu)$ at positive $\nu$ and for several values of $Q$. The solid, dashed, dotted and dash-dotted lines correspond to $\mathrm{Q}=1,2,3$ and 5 respectively 


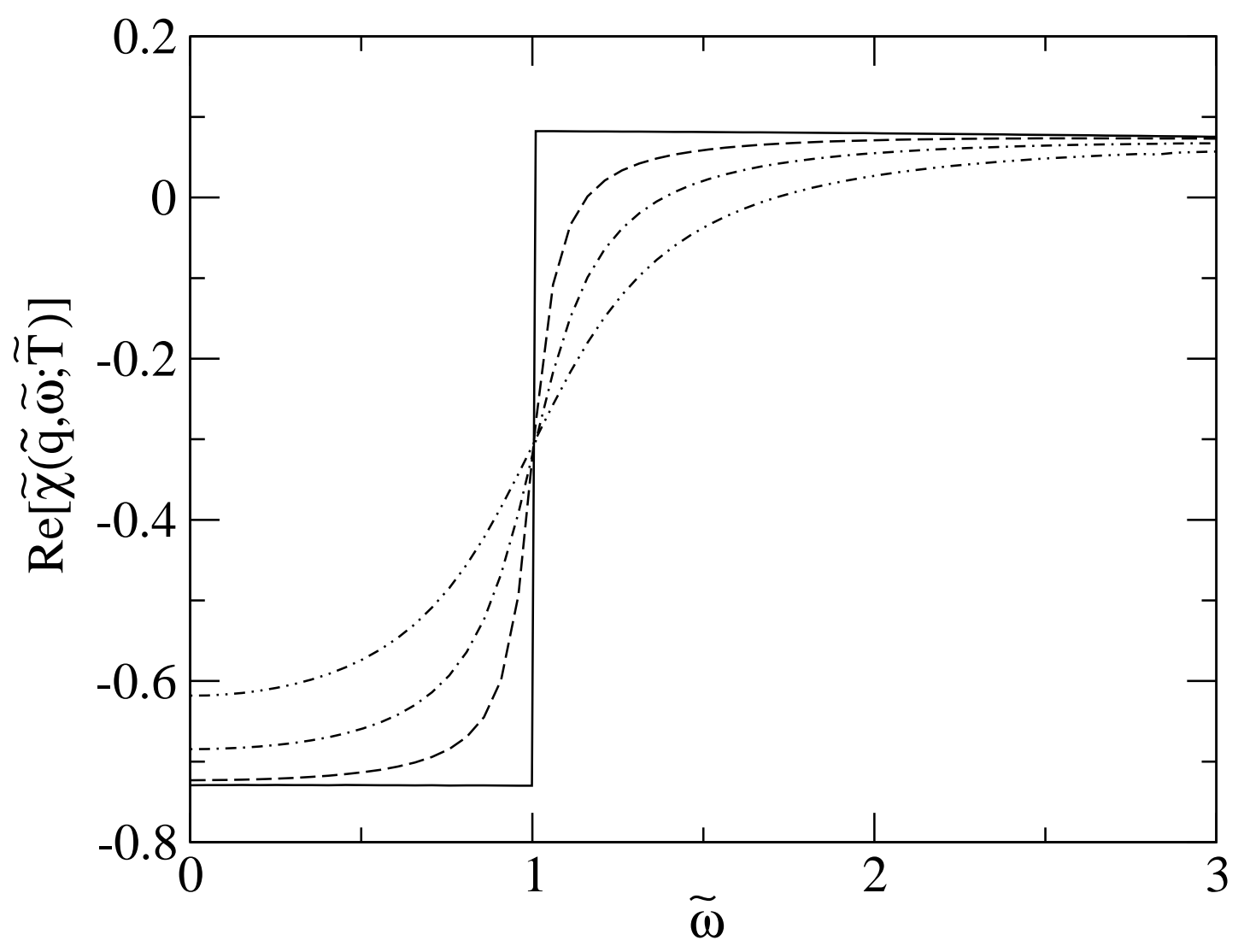

Figure 2: Real part of $\tilde{\chi}(\tilde{q}, \tilde{\omega} ; \tilde{T})$ at temperatures slightly below and above the Bose-Einstein transition temperature $\tilde{T}_{c} \approx 6.62$. Solid line: $\tilde{T}=6.5$, dashed line: $\tilde{T}=6.7$, dot-dashed line: $\tilde{T}=6.8$, and dot-dot-dashed line: $\tilde{T}=7$. 


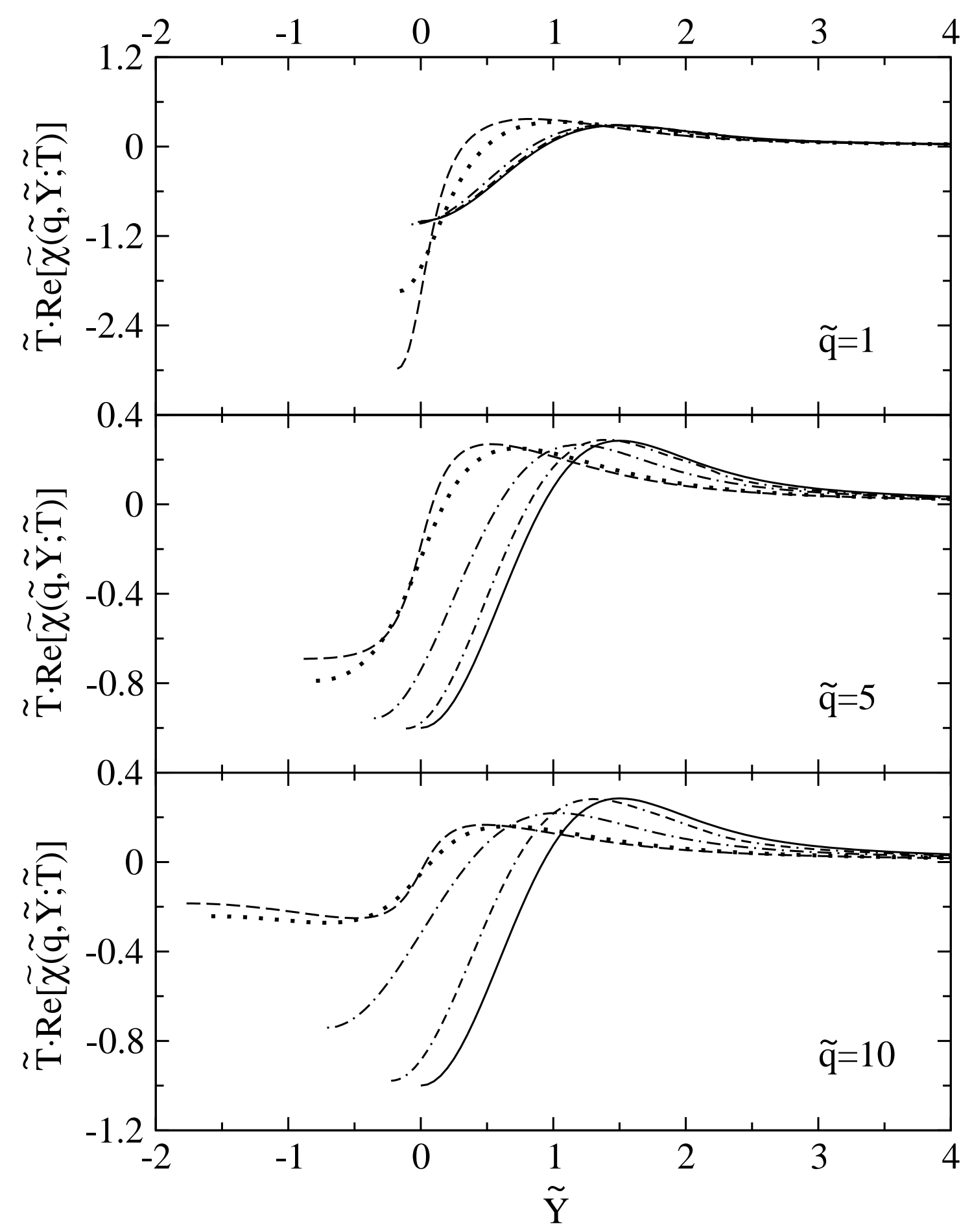

Figure 3: Evolution of $\tilde{T} \operatorname{Re}[\tilde{\chi}(\tilde{q}, \tilde{Y} ; \tilde{T})]$ with $\tilde{T}$ and for three values of the momentum transfer $\tilde{q}$. The dashed, dotted, dot-dashed and dot-dashed-dashed lines stand for $\tilde{T}=8,10,50$ and 500 respectively, while the solid line corresponds to the classical limit 


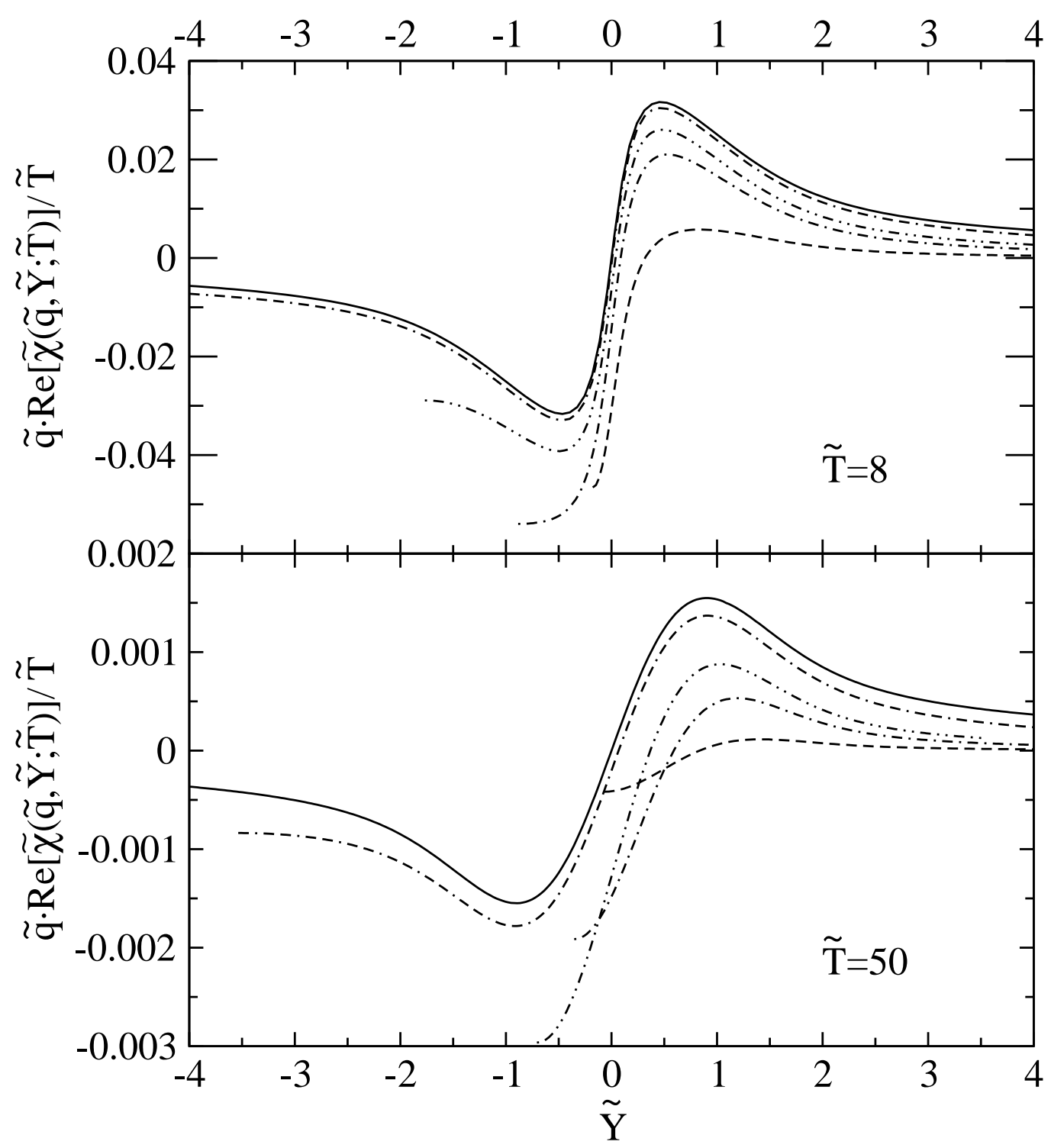

Figure 4: Momentum dependence of the $\tilde{q} \operatorname{Re}[\tilde{\chi}(\tilde{q}, \tilde{Y} ; \tilde{T})] / \tilde{T}$ at two different temperatures. The dashed, dot-dashed, dot-dot-dashed and dash-dash-dotted lines stand for $\tilde{q}=1,5,10$ and 50 respectively, while the solid line corresponds to the limit $\tilde{q} \rightarrow \infty$. 


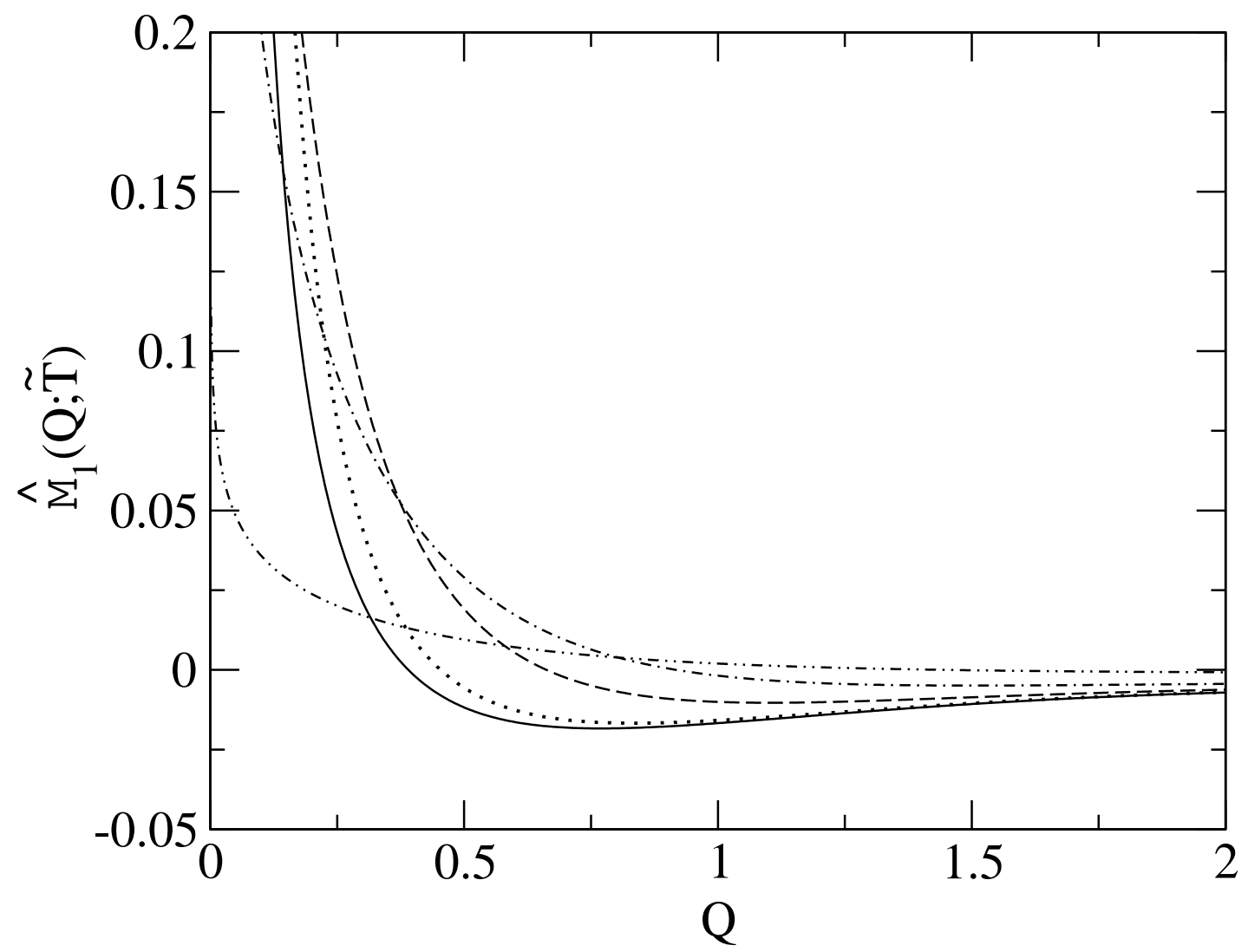

Figure 5: First moment of the real part of the dynamic susceptibility (Eq. (28)) as a function of $Q$ and for several values of temperature. The dotted, dashed, dot-dashed and dot-dot-dashed lines stand for $\tilde{T}=7,8,10$ and 25 , respectively, while the solid line corresponds to any $\tilde{T}$ below $\tilde{T}_{c}$. 